\title{
Assessment and recommendations for a consolidated European approach to space weather - as part of a global space weather effort
} \author{
and Manuela Temmer ${ }^{12}$ \\ ${ }^{1}$ Department of Physics, Umeå University, 90187 Umeå, Sweden \\ 2 Institute of Experimental and Applied Physics, Kiel University, 24118 Kiel, Germany \\ ${ }^{3}$ Royal Observatory of Belgium Brussels, 1180 Uccle, Belgium \\ ${ }^{4}$ National Observatory of Athens, 11851 Athens, Greece \\ ${ }^{5}$ Rutherford Appleton Laboratory, OX11 0DE Didcot, UK \\ ${ }^{6}$ Department of Physics and Technology, University of Bergen, 5007 Bergen, Norway \\ ${ }^{7}$ Finnish Meteorological Institute, 00101 Helsinki, Finland \\ ${ }^{8}$ Department of Physics and Astronomy, University of Leicester, LE1 7RH Leicester, UK \\ ${ }^{9}$ University Grenoble Alpes, CNRS, IPAG, 38000 Grenoble, France \\ ${ }^{10}$ INAF Astronomical Observatory of Trieste, 34131 Trieste, Italy \\ ${ }^{11}$ Department of Physics, University of Trieste, 34127 Trieste, Italy \\ 12 Institute of Physics, University of Graz, 8010 Graz, Austria
}

Hermann J. Opgenoorth ${ }^{1,8, *}$, Robert F. Wimmer-Schweingruber ${ }^{2}$, Anna Belehaki ${ }^{4}$, David Berghmans ${ }^{3}$, Mike Hapgood ${ }^{5}$, Michael Hesse ${ }^{6}$, Kirsti Kauristie ${ }^{7}$, Mark Lester ${ }^{8}$, Jean Lilensten ${ }^{9}$, Mauro Messerotti ${ }^{10,11}$,

Received 25 July 2019 / Accepted 30 August 2019

\begin{abstract}
Over the last 10-20 years there has been an ever-increasing international awareness of risks to modern society from adverse and potentially harmful - and in extreme cases even disastrous - space weather events. Many individual countries and even international organisations like the United Nations (UN) have begun to increase their activities in preparing for and mitigating effects of adverse space weather. As in the rest of the world there is also in Europe an urgent need for coordination of Space Weather efforts in individual countries as well as in and among European organisations such as the European Space Agency (ESA) and the European Union (EU). This coordination should not only improve our ability to meet space weather risks, but also enable Europe to contribute to on-going global space weather efforts. While space weather is a global threat, which needs a global response, it also requires tailored regional and trans-regional responses that require coordination at all levels. Commissioned by the European Space Science Committee (ESSC) of the European Science Foundation, the authors - together with ex-officio advice from ESA and the EU - have over two years assessed European activities in the realm of space weather and formulated a set of recommendations to ESA, the EU and their respective member states, about how to prepare Europe for the increasing impact of adverse space weather effects on man-made infrastructure and our society as a whole. We have also analysed parallel international activities worldwide, and we give advice how Europe could incorporate its future activities into a global scheme.
\end{abstract}

Keywords: space weather / hazards / societal effects / public Issues / strategy

\section{Process}

This report was commissioned by the European Space Science Committee, ESSC, of the European Science Foundation, ESF, in May 2017. The authors of this short version of the full report (which is appended as Supplementary Material) are the expert members of the European Space Weather Assessment and Consolidation Committee (ESWACC).

\footnotetext{
*Corresponding author: hermann. opgenoorth@umu. se
}

Ex-officio advisors were Juha-Pekka Luntama, ESA-SSA (European Space Agency - Space Situational Awareness), ESOC (European Space Operational Center), Germany, Mats Ljungqvist, EC-DG (European Commission - Directorate General) Growth, Brussels Belgium, and Georg Peter, EC/Joint Research Centre, Ispra, Italy.

(NOTE: the ex-officio representatives are not co-authoring this report, but have given valuable comments and advice throughout the process of ESWACC meetings and discussions). 
Starting with a first meeting at ESOC in Darmstadt in June 2017, ESWACC has held four face-to-face meetings, at ROB in Brussels, at ESA-ESTEC (European Space Research and Technology Centre) in Noordwijk, and at the UN in Vienna.

This report was completed following a number of teleconferences also to prepare slides with preliminary findings and recommendations as requested by the EU/DG-Growth in February 2018 and another set of slides with the final ESWACC findings and recommendations for the Director General (DG) of ESA, Dr. Jan Wörner at ESA Headquarters (HQ) in February 2019.

The next-to-final report was presented to the ESSC Plenary Meeting in Amsterdam on May 9-10, and was finalised after further iteration and helpful comments from Profs. Dan Baker, LASP, Boulder, USA, Ian Mann, U. of Alberta, Canada, Tim Fuller-Rowell, CIRES, U. of Colorado and NOAA, USA, and Karel Schrijver, Lockheed Palo Alto (retired), USA.

\section{Executive summary}

Over the last 10-20 years there has been an ever-increasing international awareness of risks to modern society from adverse and potentially harmful - and in extreme cases even disastrous space weather events. Many individual countries and even international organisations like the United Nations (UN) have begun to increase their activities in preparing for and mitigating effects of adverse space weather. As in the rest of the world there is also in Europe an urgent need for coordination of Space Weather efforts in individual countries as well as in and among European organisations such as the European Space Agency and the European Union. This coordination should not only improve our ability to meet space weather risks, but also enable Europe to contribute to on-going global space weather efforts. While space weather is a global threat which needs a global response it also requires tailored regional and trans-regional responses that require coordination at all levels.

This report discusses on-going European SWx (in the following we will adopt the US standard abbreviation "SWx" for Space Weather) efforts and issues, and gives recommendations for future coordinated and better consolidated activities. We have found that these issues can be broken down into six activities where coordination at European level will be required.

This report makes recommendations in these six areas - as defined by:

Area 1. Enabling critical science to improve our scientific understanding of $S W x$ :

Our overall description of the coupled Sun-Earth system in the space age still contains critical gaps in the scientific understanding of several mechanisms through which space weather couples from space all the way down to Earth. While significant progress can and will be made using existing scientific infrastructure including existing multi-spacecraft missions and ground-based networks, support must urgently be provided for the next generation space missions and the replacement of ageing ground-based infrastructure.

Area 2. Development and coupling of advanced models by applying a system-science approach which utilises physicsbased modelling:
Develop better physics-based models and also define metrics that facilitate assessment of different models and to encourage their transition to operations.

Area 3. Assessment of risks at National, Regional and European levels:

European States should regularly assess their exposure to SWx risks and coordinate and combine their studies at regional and European level to cover the interdependency of technological infrastructures. This requires close cooperation between decision makers, SWx scientists, service providers, and end-users.

\section{Area 4. Consolidation of European User Requirements:}

European SWx user requirements should be (re-)assessed and prioritised taking into account regional and societal differences and needs, also addressing different needs of various infrastructure systems. This should be done on a regular basis, e.g., every five years, also to facilitate the exchange of information among European SWx actors.

\section{Area 5. Support to $R 2 O$ (Research to Operations) and $O 2 R$ (Operations to Research):}

The best available knowledge and models should be used in future SWx service organisations. Such transition from Research to Operations should be guided by teams of scientists all over Europe - following the distributed ESA Expert Service Centre approach. In addition, a structure to enable models that have been transitioned to operations to be improved (O2R) should be established.

Area 6. Define and implement an operational network for future SWx observations:

Based on our present scientific understanding and the above assessments of risks and user requirements we need to define an operational space- and ground-based network that measures essential space weather parameters which in turn can drive the SWx predictions required to protect our society's infrastructure.

\section{Other issues requiring attention}

A first analysis of our knowledge, observational gaps and requirements for an appropriate SWx warning system with special consideration of European SWx vulnerabilities and weaknesses, but also taking into account European strengths has been carried out by the Expert Groups in the ESA SSA Space Weather Service Network and the results of the analysis have been reviewed by the European Space Weather Working Team. However, continuous elaboration of the analysis including assessment of space weather risks on European infrastructure and understanding of the user needs will be required because of the constantly evolving end user landscape and European SWx competencies.

We find that the presently ongoing SWx efforts in Europe are to a large degree uncoordinated and also mostly unsustainable. This is probably at least partially due to the fragmentation of funding responsibilities in Europe. Apart from the ESA and the EU, individual states and many different agencies also fund space weather activities. 
The ESA is presently developing pre-operational SWXservices in the framework of its Space Situational Awareness Programme with 19 out of ESA's 22 Member States participating in the SWx segment. However, the ESA SSA programme is optional and the participating member-states contribute very diverse voluntary annual contributions, not always reflecting Net National Income. Also the scope of the services, established within this programme, is currently limited to testing, verification and validation.

The EU had - and still has - scattered H2020 and Framework Programmes (FPx) SWx calls, reoccurring every other or sometimes even only every third year. Even if the EU funding to SWx activities adds up to a considerable amount of approximately $60 \mathrm{M}$ over the last 10 years, the funding offered in each call is sub-critical to develop sustainable science and service activities, and did not match the European needs. Many of these calls were (and still are) aimed primarily at the prototyping of services with relatively little regard for the scientific foundations, which are required for such services to become reliable. Most of the work required for the scientific underpinning of SWx, especially the science and data exploitation activities (see our findings below), fall into the general EU-calls, where they compete with basic science.

Additional funding provided by individual European states is fragmented, localised, un-coordinated, and also mostly insufficient to satisfy the growing societal needs, for both the advancement of knowledge and the provision of services. Also it is difficult to build transnational and regional efforts on national funding.

Moreover, the private sector is recently becoming more and more active in space, and realises its exposure to SWx-threats. However - and yet again - the funding emerging from such sources is often too directed and topically far too narrow to satisfy SWx needs.

We would like to stress that while this diversity in funding is currently often seen as a European weakness, it could be turned into a strength, if it were coordinated according to the principle "Let those do the work who are best at it". We strongly advocate a dedicated Europe-wide coordination of SWx activities. This could be done in a similar manner to how the COPERNICUS programme deals with Earth Observations.

Current European SWx services rely on data from ageing infrastructure such as ESA's SOHO spacecraft, which is rapidly approaching its 25 th anniversary, having thus substantially exceeded its design lifetime of two years. While ESA is indeed discussing with the US about a coordinated development of a common space weather monitoring system, there is no consolidated plan yet for the successor for SOHO. The current scientific space infrastructure is not able to provide near-real time 24/7 operational data for future SWx warning systems.

The presently available fleet of space- and ground-based assets, which observe the sun, geospace, and the region of space between these two, the inner heliosphere, offer the current and unique opportunity to increase our scientific understanding of SWx. As this infrastructure continues to age beyond its operational lifetime, and given the increasing funding pressures, progress in the scientific understanding of SWx can, and needs to be made now, requiring adequate, coordinated, and reliable funding.
Europe is, of course, not alone in its recognition of the importance of SWx science and services. European agencies and researchers have long recognised the importance of international coordination of scientific efforts, information exchange on space weather events and their mitigation, national, regional and over-regional risk analysis and assessment of user needs. This coordination should be understood to encompass the currently scattered, uncoordinated, short-term, and often insufficient funding of European SWx activities and should lead to an increase in funding of SWx activities to a level that allows an operational European SWx system to be established, operated and maintained. These activities should cover both the scientific foundations of SWx as well as the development and provision of SWx services for society.

\section{Background}

The European Space Sciences Committee (ESSC - http:// www.essc.esf.org) of the European Science Foundation (ESF) is an independent committee that regularly provides expert advice to European and National research funding and research performing organisations that support space sciences in Europe.

In response to such growing interest and the increasing international awareness about potential threats from adverse space weather to our modern society the ESSC has both been asked and decided by itself to look into a consolidated advice concerning a European approach to Space Weather risk assessment and parallel scientific and service activities, by creating the European Space Weather Assessment and Consolidation Committee, ESWACC.

The aim of this new Committee was to prepare detailed recommendations for a consolidated and strategic approach to SWx - for Europe as a whole and also as a part of the global SWx effort as advocated by the UN-COPUOS (Committee on the Peaceful Uses of Outer Space).

The situation of SWx today is that we do understand the underlying principles of Sun-Planet interactions, but we are still far from an operational system for SWx predictions as we know them from terrestrial weather forecasts. A promising approach to develop the required future European space weather activities and services can best be described as an iterative loop, in which there should exist a continuous iteration and feedback between:

(a) New improved science understanding and supporting observations,

(b) Evolving requirements of European end-users and infrastructure providers, and

(c) Improved potential to deliver SWx products (based on recent science findings), where b) and c) should also address particular national and trans-national requirements, and eventually feedback into new challenges for the science efforts under a).

\section{Space weather as a global challenge}

Following the approach defined by the Committee on Space Research (COSPAR) and International Living With a Star 
(ILWS) SWx Road-Map document (Schrijver et al., 2015) the effects of space weather on technological infrastructure can broadly be defined in terms of different impact pathways:

- Geomagnetically induced currents, impacting on power and transport infrastructures;

- Radiation effects leading to ageing and malfunctions of space, aviation and in severe cases even ground assets, including direct impacts on radio wave and other communication transmissions;

- And combined effects of both radiation and current flow effects, leading to disturbances of navigation and communication systems, increased satellite drag and thus decreased satellite lifetime, as well as inaccurate assessments of satellites' orbits, which in turn could increase collision risks with space debris, as a secondary SWx impact.

\section{Findings and recommendations}

The challenges stated above, imposed by the SunHeliosphere-Geospace system itself, imply the following basic and overarching principles of a future European SWx architecture - yet to be developed:

- Predictions of space weather events require a deep understanding of the underlying science and rely on the availability of a variety of different - often scientific - data sets. These data sets need to be combined, modelled, analysed, and assimilated into networks of models and datasets. This complex flow needs to be constantly improved.

- Observations and the processing of corresponding and other relevant data from Sun to Earth must be available in near real time.

- Coordinated observing capability between all space- and ground-based monitors must be improved.

- Regional ground-based networks of sufficient station density need to be coupled to allow the understanding, now-casting, and prediction of - amongst others - magnetospheric disturbances (substorms and spikes in storms) and ionospheric irregularities (scintillations, sporadic E layers, travelling ionospheric disturbances) that have mostly regional impacts.

- Instruments for observing the upper atmosphere, magnetosphere, heliosphere and the sun need to be calibrated and inter-calibrated, to enable both long-term observations and intercomparability. This recurring activity requires coordination and oversight.

- Standardised procedures for data archiving, preservation and open access need to be developed and implemented. This also applies to models and model results.

- Modelling of SWx phenomena needs a very strong effort on the physical interactions and feedback mechanisms in the Sun-Earth system in a coordinated, and coherent manner.

- Such modelling should cover not only the Earth-Sun system, but also the inner heliosphere to allow better validation by using measurements by existing and future deep space missions and to support (and prepare for human) exploration of the solar system.

\subsection{Area 1: Imminent need for critical research with dedication to enable space weather understanding and prediction}

A science-driven approach to the mitigation of space weather effects increases confidence in the assessments of risk and socio-economic impacts and in the accuracy of their results. As discussed in the COSPAR/ILWS roadmap (Schrijver et al., 2015), and despite very significant recent improvements in the understanding of the drivers of space weather, scientists are still a long way from being able to offer high quality forecasting of impending severe space weather that provides concrete benefits to users.

The complexity of SWx phenomena has led to a distinction being made between high quality forecasting and high precision forecasting. SWx forecasts need to distinguish between amplitude and timing of the phenomena, especially because the ubiquitous process of magnetic reconnection, which underlies much of SWx, is highly unlikely to be predictable in the deterministic sense. While high precision probabilities of the amplitudes of such events may be possible in the future, we do not expect to be able to predict their onset times in the coming decades. We may ultimately have to embrace uncertainty as a fundamental part of space weather forecasting.

\section{Findings}

Research in the past decades has led to substantial progress in our understanding of the overall big picture of the Sun-Earth SWx chain. The network of space- and ground-based assets, which formed the basis for our present scientific understanding of the SWx chain, is ageing and in danger of turning insufficient to provide the reliable input needed for a realistic operational SWx system. At present there is no indication that we will have a similar fleet of spacecraft at our disposal in the near future. Networks of ground-based stations are presently operated on decreasing, often uncoordinated and unstable national or even institutional budgets. Nevertheless, most present-day Space Weather services rely on such networks, which may suddenly disappear with a loss of their funding.

At the European level, we lack a coordinated effort to combine the results of past, ongoing, and future SWx-enabling research into a SWx prediction framework. We also lack a coordinated European science effort to combine the results of individual SWx-enabling research activities.

The "Bureaucracy Load" presently existing at both ESA-SWE (Space Weather) and the EU H2020 grant and project management is very high especially for university science leaders. ESA, and to same extent the EU, need to recognise that such leaders of science teams are generally directly involved in the scientific work of the studies funded by ESA and the EU. Unlike in industry, there is not a separate layer of management in scientific institutions. The contract management process for science teams needs to reflect this, striking a balance between that process and the need to make scientific and technical progress. Science leaders need time to do their scientific work between project meetings and reporting. A failure to respect that will divert already scarce resources away from scientific work and is likely to delay technical and scientific progress. 


\section{General recommendations for Area 1}

1. Sustained and adequate financial support to a directed SWx-enabling research effort in solar, heliospheric and magnetospheric/ionospheric/atmospheric physics to build a better knowledge base for future SWx services.

2. Exploitation of existing SWx datasets for near Geo-space (e.g., SWARM and other LEO and GEO satellites, ...), in the magnetosphere (e.g., Cluster, Themis, MMS, Van Allen Probes, ...), in the Heliosphere and for the Sun (e.g., SOHO, ACE, Wind, SDO, Hinode, Parker Solar Probe, ...) should be lifted out of future general H2020 data exploitation calls by creating a "dedicated funding line" for space weather enabling science, with the declared goal to improve SWx forecast reliability and in order to stimulate the "harvesting of low hanging fruits"!

3. Efforts to combine data and results from connected regimes (system science) should be encouraged.

4. Any such funding should be on a long-term and regular, and continuous basis to make best use of the European assets.

5. Foster collaboration both within Europe and with similar efforts around the world.

\section{Additional recommendations with respect to ground-based efforts:}

1. Dedicated and, if at all possible, coordinated financial support, both at national and European level, to groundbased network efforts which support SWx-enabling research in the physics of the Sun, heliosphere, magnetosphere, ionosphere, atmosphere, and solid Earth physics in order to build a better knowledge base for future SWx services. This support is needed for the continuation and maintenance of such networks and to close gaps.

2. Encourage member states to see funding of national instrument assets (often parts of networks) as a global subscription for access to all data from wider European and global programs of similar instruments and as a critical national contribution to a wider and important pan-European SWx observing network.

3. Efforts should be supported to combine and coordinate any European assets concerning such instrumentation into regional and global networks, to enable improved collaboration and data-sharing with other space-based SWx assets.

4. It is important to decrease the "Bureaucracy Load" presently existing at both ESA-SWE and the EU H2020 grant and project management.

\subsection{Area 2: Support to system-science approach with coupled physics-based modelling: Sun/solar wind/magnetosphere/ionosphere/atmosphere}

Any scientific effort based on observations must inevitably be complemented by the development of physics-based models to explain, or eventually even predict relevant observations in plasma regimes addressed in this subsection. Predictive models are therefore critical to a robust forecasting capability, which provides workable reaction times to parties affected by space weather. In addition, our ability to successfully predict the space environment and space weather phenomena constitutes a measure of the level of our scientific understanding.

\section{Findings}

Today's understanding of the physics of the Sun, the solar wind and the magnetosphere/ionosphere/atmosphere system is incomplete and does not allow the reliable predictions, which are needed for operational purposes.

Even the most advanced models at our disposal have critical shortcomings and gaps, especially in coupling the immense diversity of the associated physical scales, as illustrated in Section 3.1.

Most present day empirical models suffer from such drawbacks, but are nevertheless important for immediate and cost-effective progress in space weather predictions mostly through parameterisation, and remain of value for verification/validation of other models. This translates into an urgent need for further development of advanced physics-based models and also to couple such models for various regimes in space with each other. It will be essential to run such models in ensemble mode to evaluate and quantify forecast uncertainties.

Only then will we be able to make sufficiently accurate forecasts of timing, location and severity of SWx effects in the coupled solar-terrestrial system, which are essentially required to protect our technological assets.

\section{Recommendations for Area 2}

1. There should be a dedicated and sustained financial support in Europe for the development of state-of-the-art physics-based models for the Sun, the solar wind and the magnetospheric/ionospheric/atmospheric/solid Earth system. The funding responsibilities need to be transparent to SWx researchers, developers, and users.

2. Scientific groups carrying out such overarching model efforts require a certain critical mass to be able to both digest scientific findings and implement those into advanced models. We recommend to look into new funding models to support such overarching model efforts and create groups with critical mass.

3. A periodic, e.g., triennial, review of development success and recommendations for future developments and investments should be implemented.

4. In order to define and monitor future progress of such efforts one will need to develop a mechanism together with space weather user groups - also using well defined scientific and operational metrics - to verify/validate and compare the performance of physics-based models, both throughout Europe and in collaboration with other global efforts.

5. Using the NASA Community Coordinated Modelling Centre, CCMC, as a role model for a test-bed of coupled predictive models, coordinated European efforts (like e.g., the Virtual SWx Modelling Centre, VSWMC) should be supported to combine and couple state-of-theart models into an operational chain of predictive models from the Sun to the Earth. 


\subsection{Area 3: Consolidation of national, regional and European risk assessments}

Space weather risks constitute an ever-evolving landscape. The successful mitigation of these risks requires a detailed and continuing assessment of SWx impact pathways, of the risks that flow from these pathways, and of the options to mitigate these risks. Only when mitigation options are known is it possible:

(a) To assess the socio-economic value of taking action (i.e., does mitigation cost significantly less than the economic loss), and thus,

(b) To establish requirements for mitigation including both system hardening and space weather forecasting.

Space weather risks are continuously evolving with the advance of science and technology. This is shown by the evolution of space weather impacts on technological systems since the first recorded impacts on electric telegraph systems, then through impacts on telephones, radio communication and power grids.

\section{Findings}

At present we lack a complete and true evaluation and description of European SWx risks for most individual countries and particularly for regional and over-regional risks, which are emerging from the increasing interdependencies and interconnections of the potentially affected infrastructure.

The risks emerging from SWx events - and thus the definition of user-requirements - will obviously not be the same for different parts of Europe or for European activities in other parts of the world or in space. Any such risks are also different for space and ground-based technological assets.

There is an urgent need for a coordinated European assessment of national and regional risks and consequent potential socio-economic impacts of a variety of space weather events, both for extreme and average daily solar activity.

Only on the basis of such a risk assessment a full catalogue of European User Requirements can be compiled for the development of a future European space weather service function.

\section{Recommendations for Area 3}

1. Encourage and enable member-states to carry out a coordinated Europe-wide effort of national risk and socio-economic impact studies of SWx events - in close collaboration between SWx scientists and engineers working with potentially affected infrastructure.

2. Support the combination and expansion of national risk assessments into regional and Europe-wide risk and impact analyses, addressing the interdependency and connectivity of many - if not all - technological infrastructures in Europe - build on laudable efforts already conducted by the EC-JRC (Joint Research Center) and in line with the EU mandate for coordination of such risk assessments (as provided by the Union Civil Protection Mechanism). We strongly recommend that space weather be included in the next iteration of the risks to be addressed by the JRC.
3. Support and enable awareness about and the dissemination of such risk assessments to national decision makers, but also to the communities of scientists, service providers and end-users alike.

4. Create an active exchange forum for tri-lateral discussions and regularly updated information exchange between SWx Scientists, End Users and Service Providers. The annual "European Space Weather Week", ESWW, serves as a good model.

\subsection{Area 4: Consolidation of European user requirements}

ESA carried out extensive studies of space weather service requirements in the first decade of this century. Two parallel studies in 2000/2001 provided comprehensive analyses. These studies were consolidated during the first period of the ESA Space Situational Awareness programme, producing the requirements documents on which ESA has built the space weather element of its SSA programme.

Informal discussions, with ESA and across the wider community, have noted the need for continued elaboration of space weather requirements to reflect subsequent changes in space weather risks, and in our understanding of those risks. Such improvement must provide a clear and comprehensive prioritisation of requirements, addressing the spread of space weather risks across critical European infrastructures on the ground as well as in space, and engaging with the space weather risks identified by civil protection authorities across Europe. This is a challenging task as it requires a process (and sufficient resources) to engage with the specific needs of individual European countries and then to consolidate those into a coordinated and prioritised programme.

\section{Findings}

At present we require results from the full European-wide risk assessment as described in Section 3.3 to elaborate and complement the customer requirements compiled by the ESA SSA programme.

ESA SSA requirements have been the baseline that was used to develop the current network of SWx Expert Centres and Expert Groups, but a continuous elaboration of the requirements will be needed to take into account the continuously evolving user landscape and scientific knowledge base.

Continuous elaboration of the understanding of the user needs for tailored services providing actionable information is mandatory. At present this is carried out only in the framework of the ESA SSA Programme with limited resources.

Only on the basis of a full Europe-wide risk assessment as described in Section 3.3 can a new and complete catalogue of European User Requirements be compiled, addressing risks on all assets. In this context we note that the European space assets comprise only about $10 \%$ of all assets at risk from SWx. It is also critical that this compilation is updated typically every five years.

The catalogue of user requirements should be a "living document" with update intervals of e.g., every five years, since the infrastructures and their particular vulnerabilities and resiliencies constantly change and develop - as does the 
scientific understanding of potential SWx events and their associated risks.

\section{Recommendations for Area 4}

1. Support and enable a coordinated Europe-wide effort to elaborate European SWx user requirements based on needs as specified by local region (such as arctic, subauroral, mediterranean) and infrastructure domain (communication, energy, health, finance, etc.), addressing the risks from SWx impacts on space-based and groundbased infrastructure - building on efforts already conducted by the ESA SWE Programme and the EC-JRC and re-establish SWx as a task for the JRC activities.

2. There will be a need to prioritise user requirements in the catalogue, based as far as possible on their value in mitigating particular impacts, e.g., specifically forecasts helping operators to maintain power supplies, navigation or communication.

3. Enhance the exchange and updating of information about user requirements in tri-lateral discussions between SWxScientists, End-Users and Service Providers. For most requirements the description of context and clear rationale for the expected impact will be crucial to determine the needed forecast type and quality.

\subsection{Area 5: "R2O" and "O2R" or how can SWX scientists interface with candidate organisations for SWX services - in Europe and globally}

Unlike terrestrial weather, which is a mature science, space weather is in its scientific infancy. The immature nature of the field, the complexity of data sets, and the rapidly evolving character of models make the close involvement of active researchers highly beneficial to space weather services. Practising scientists can distinguish a data glitch from valid data, and valid model output from erroneous results. Furthermore, our data sources are sparse and often ephemeral, a situation which mandates that we use any available information to form the best possible picture of space weather and to produce the best possible forecasts.

We thus need a flexible approach, where every institution and country can participate to the best of their abilities, and which combines scientific and interpretative skills at many institutions. Such an inclusive model also provides rapid utilization of emerging capabilities and knowledge. Finally, it supports decision-making based on knowledge of what is possible today and what is emerging in the research community.

\section{Findings}

There is no doubt that initial SWx services and predictions of events and effects are urgently needed today by a variety of end users and decision makers to protect their assets in space and on the ground - but the present SWx knowledge base is insufficient for this task.

The development of future improved SWx services must be driven by specific User Requirements (see Sect. 3.4), and must be based - and constantly improved - on the basis of a thorough SWx Risk Assessment (see Sect. 3.3).
Future services should continuously be improved on the basis of the best available scientific knowledge - including making use of the best performing, coupled and "state-of-the-art" models available.

A promising approach to develop the required future European space weather activities and services can best be described as an iterative loop between $\mathrm{R} 2 \mathrm{O}$ and $\mathrm{O} 2 \mathrm{R}$ in which there should exist a continuous iteration and feedback between:

(a) New improved science understanding and supporting observations,

(b) Evolving requirements of European end-users and infrastructure providers, and

(c) Improved potential to deliver SWx products (based on recent science findings),

where b) and c) should also address particular national and trans-national requirements, and eventually feedback into new challenges for the science efforts under a).

To this end a constant round-table of dialogue between all SWx partners, i.e., SWx scientists, European policy makers, public and private SWx service organisations and representatives from the user communities will be required at such discussions.

The decentralised ESA SSA approach of distributed and networked SWx Expert Service Centres for various parts of the coupled Sun-Earth system (still under development), is a promising approach towards the development of future European Space Weather Prediction Centres - both fulfilling the above requirements and making the best use of the distributed European expertise and capabilities.

\section{Recommendations for Area 5}

A European SWx effort should:

1. Utilise and coordinate existing national efforts to provide regional space weather services (Examples: Belgium: ROB $^{1}$ \& VSWMC ${ }^{2}$, UK: Met-Office, France: OFRAME ${ }^{3}$, Italy: $\operatorname{INSWSN}^{4}$ )

2. Involve ESA, EU/EC and all member-states, and must be driven by the European user requirements, as discussed in Section 3.4.

3. Be conducted also in close coordination and cooperation with the already ongoing parallel developments on a global scale (UN-COPUOS), and with efforts of other nations (US, China, Russia, Japan, etc.)

4. Benefit from the experience in the development of global services and 24/7 operations of existing global organisations such as $\mathrm{WMO}^{5}, \mathrm{ICAO}^{6}, \ldots$

5. Involve expert scientists in the development of future European SWx services so these services become and remain competent in the plasma-physical context of the Sun-Earth System.

${ }^{1}$ ROB: Royal Observatory of Belgium.

${ }^{2}$ VSWMC: Virtual Space Weather Modeling Centre.

${ }^{3}$ OFRAME: Organisation Française pour la Recherche Applicative en Météorologie de l'Espace.

${ }^{4}$ INSWSN: INAF National Space Weather Service Network.

${ }^{5}$ WMO: World Meteorological Organisation.

${ }^{6}$ ICAO: International Civil Aviation Organisation. 
6. Build on and expand the present decentralised and distributed ESA-SWE Expert Service Centre (ESC) approach as a good example for such efforts.

7. Update the resulting European User Requirement Document on a timescale of 3-5 years - to adapt to emerging needs of the user community.

\subsection{Area 6: Define and implement a network of space and ground-based assets for future SWX observations}

Currently space-weather related activities benefit enormously from a unique and existing fleet of scientific spacecraft observing the sun, the heliosphere and geo-space, supported by a multi-facetted network of complementary ground-based measurement infrastructure. These assets can still support most of the international efforts to drive forward and support an improved knowledge in the science of space weather processes (see our findings in Sect. 3.1). While unique in its kind, the present fleet of science-oriented spacecraft in geospace and the heliosphere is nevertheless ageing and obviously it also does not fulfil the requirements of a future operational system for space weather services.

\section{Findings}

Based on the emerging scientific understanding of space weather events and processes, scientists and space agencies together will have to define an essential and optimum set of observable parameters at the Sun and in the heliospheric, magnetospheric, ionospheric, atmospheric, and solid-Earth system, which are needed to characterise the energetic and dynamical state of the most important elements of the Sun-Earth coupled plasma regimes and to drive the required forecasts of the expected response of the system as a whole.

Based on such required sets of observables one will have to define both a baseline and an optimum network of space and ground-based instrumentation, which can monitor such required parameters with sufficient accuracy, 24/7 and in realtime.

We stress, however, that any future observational network must be able to do (a little) better than the bare minimum SWx requirement and - by design - always also allow new science to emerge in order to prepare for potentially growing future SWx user requirements.

Any future European network for space weather observations in space and on the ground should also be embedded in and be coordinated with a global effort of other agencies and nations - coordination implies both complementarity and comparability in terms of location, type of measurement, and inter-calibration. The planned ESA Lagrange mission to L5 for improved solar and solar wind monitoring in concert with other international efforts at L1 is a good example for such coordination.

However, there will still be a need for additional purely scientific and space weather enabling missions for an increased understanding of the processes in the Sun Earth system. This effort cannot be replaced by $24 / 7$ operational missions, in particular not if they are aimed at the observations of essential SWx-parameters or proxies only.

\section{Recommendations for Area 6}

Directed towards ESA:

1. Create a forum between SWx scientists, staff of the ESA-SSA and ESA-SCI (Science) programmes and the space agencies of individual member states for the definition and future operation of a "fleet" of dedicated spacecraft: large crucial cornerstone missions, small satellites at key locations and hosted payload elements for European Space Weather purposes.

2. For this dedicated "fleet" of SWx space assets define a set of observables, fulfilling the present user requirements and at the same time allowing for future science development to allow for an increased knowledge base for future emerging SWx requirements.

Directed towards EU, Member States, Funding and Civil Protection Agencies:

1. On the basis of such considerations and in collaboration with individual member states support the maintenance, modernisation and future augmentation of ground-based instrument networks for space weather purposes to support the space assets for SWx observations.

2. Each type of mission, payload or ground-based instrument network should become part of a specific type of European and/or global network:

- Examples of network "types": Solar observations, solar wind observations at L1/L5, and various geo-space regimes: magnetosphere, ionosphere, atmosphere, ring current.

- Present ground-based instrument networks comprise: Magnetometer networks and coordination through SuperMAG, coherent scatter radar systems SuperDARN ${ }^{7}$, GNSS $^{8}$ receivers, Ionosondes, Incoherent scatter radars like EISCAT ${ }^{9}-3 \mathrm{D}$, solar radio-observations, LOFAR $^{10}$, $\mathrm{GONG}^{11}, \mathrm{NMDB}^{12}$, etc.

NOTE: All data in this combined space and ground-based network of SWx assets should be collected and disseminated under an Open Data Policy - and for potential parallel scientific use it should even include the original raw and S/C housekeeping data.

\section{Conclusions, overarching recommendations and future prospects}

We have argued that - as in the rest of the world - there is also an urgent European need for coordination of space weather efforts in individual countries as well as in and among European

\footnotetext{
${ }^{7}$ SuperDARN: Super Dual Auroral Radar Network.

${ }^{8}$ GNSS: Global Navigation Satellite System.

${ }^{9}$ EISCAT: European Incoherent Scatter Scientific Association.

${ }^{10}$ LOFAR: Low-Frequency Array.

${ }^{11}$ GONG: Global Oscillation Network Group.

12 NMDB: Neutron Monitor Data Base.
} 
organisations, such as the European Space Agency and the European Union. This coordination should not only improve our ability to meet space weather risks, but also enable Europe to contribute to on-going global space weather efforts. While space weather is a global threat which needs a global response it also requires tailored regional and trans-regional responses that require coordination at all levels.

We have shown that there are six essential and indispensable activities, which urgently require coordination at European level:

1. Enabling critical science to improve our scientific understanding of SWx,

2. Development and coupling of advanced models by applying a system-science approach which utilises physics-based modelling,

3. Assessment of risks at national, regional and European levels,

4. Consolidation of European User Requirements,

5. Support to R2O and O2R,

6. Define and implement an operational network for future SWx observations.

Many countries are developing increasingly sophisticated infrastructure, which at this point can still be better prepared against SWx risks. This is a crucial step towards making our modern and technology-reliant society sustainable and resilient. Outside Europe such efforts should embrace developing nations, where such preparations are especially timely and efficient.

\section{Final recommendations:}

Europe should collaborate with other global partners (which work with different funding structures and opportunities) to reap mutual benefits and respond and contribute to parallel worldwide initiatives from global organisations as e.g., the United Nations:

- The ESA and the EU need to coordinate their efforts and share the European responsibilities for SWx activities: science and research support, observations and service as they do for other global issues, such as the Copernicus and Galileo projects.
- The ESA in particular should take care of dedicated operational SWx missions, hosted instrumentation, data production and dissemination - and continue to develop initial service functions on the basis of the presently on-going model of distributed Expert Service Centres, ESCs.

- The EU should complement ESA's efforts by stimulating and funding overarching SWx science \& data exploitation in a continuous and sustainable fashion. It should coordinate or support additional space and ground-based SWx efforts in member states or groups of member states, which are located under or at the magnetic footprints of certain space plasma regimes or in particular geographical regions and so can serve particular SWx observational requirements.

- Member-states and local groups of member-states should coordinate their national efforts to support the overarching activities of ESA/EU with respect to national and regional priorities and abilities.

- Any dedicated European SWx organisation must serve future societal needs, based primarily on European risks and consequent user needs, but in global coordination and with the continued support from the science community.

\section{Supplementary material}

Report of European Space Weather Assessment and Consolidation Committee - ESWACC (Full Version - August 30, 2019).

The Supplementary material is available at https://swscjournal.org/10.1051/swsc/2019033/olm

\section{References}

Schrijver CJ, Kauristie K, Aylward AD, Denardini CM, Gibson SE, et al. 2015. Understanding space weather to shield society: A global road map for 2015-2025 commissioned by COSPAR and ILWS. Adv Space Res 55(12): 2745-2807. DOI: 10.1016/j. asr.2015.03.023.

Cite this article as: Opgenoorth HJ, Wimmer-Schweingruber RF, Belehaki A, Berghmans D, Hapgood M, et al. 2019. Assessment and recommendations for a consolidated European approach to space weather - as part of a global space weather effort. J. Space Weather Space Clim. 9, A37. 